\title{
Inductance and Capacitance of the Multi Josephson Junction in Superconductor
}

\author{
H. M. A. R. Maruf ${ }^{*}$, M. R. Islam², F.-U.-Z. Chowdhury ${ }^{1}$ \\ ${ }^{1}$ Department of Physics, Chittagong University of Engineering and Technology, Chittagong, Bangladesh \\ ${ }^{2}$ Department of Physics, University of Chittagong, Chittagong, Bangladesh \\ Email: `hasnatmaruf@gmail.com
}

How to cite this paper: Maruf, H.M.A.R., Islam, M.R. and Chowdhury, F.-U.-Z. (2018) Inductance and Capacitance of the Multi Josephson Junction in Superconductor. Journal of Applied Mathematics and Physics, 6, 2544-2552.

https://doi.org/10.4236/jamp.2018.612212

Received: November 6, 2018

Accepted: December 15, 2018

Published: December 18, 2018

Copyright (c) 2018 by authors and Scientific Research Publishing Inc. This work is licensed under the Creative Commons Attribution International License (CC BY 4.0).

http://creativecommons.org/licenses/by/4.0/

\begin{abstract}
The characteristic behavior of the inductance and capacitance of multi junction ac Josephson effect in superconductor has been presented. Few parameters characterizing the behavior of Josephson junctions are needed to evaluate for technological applications. In this paper, the inductance and capacitance of the multi Josephson junction connected in parallel are evaluated, using simple classical argument. The numerical results for inductance and capacitance have also been included, indicating few technological applications.
\end{abstract}

\section{Keywords}

Josephson Effects, Multi Josephson Junction, Inductance, Capacitance

\section{Introduction}

The Josephson effect [1] [2] is a macroscopic quantum phenomenon in superconductivity. When a thin layer of insulating material separates two superconductors, Cooper pairs are able to tunnel through the insulator from one superconductor to the other analogous to the quantum mechanical tunnel effect. The coherence nature of Cooper pair in superconductor gives macroscopic quantum interference effect analogous to optical physics. The interference effect can be observed [3] [4] when two or more Josephson junctions (so-called multi junction) are connected together. The tunneling Cooper pair causes spontaneous supercurrent through the Josephson junction without applied voltage called dc Josephson effect and the supercurrent is proportional to the sine of the difference of phases of two superconductors. This is a very strange phenomenon in physics. As because of it, most of the physics classes preach that $V=I R$. So that without any voltage difference, there is no current. Josephson also predicted that if an external dc voltage $V$ is applied to the junction, an alternating supercurrent 
flows through the junction with frequency $\omega_{J}=(2 \mathrm{eV} / \hbar)$. This is called the ac Josephson effect. Both the $\mathrm{dc}$ and ac Josephson effects are responsible for the technological application of superconductor devices. Many superconducting devices [2] [5] [6], like SQUID (superconducting quantum interference device, consist of one or two Josephson junctions), high-frequency oscillator, Josephson voltage standards, ultrafast switching elements, Qubit in the quantum computer, Quantum Interferometers, Quantum sensing, Imaging etc., have been developed based on these effects.

A model of the multi junction ac Josephson effect in superconductor has been proposed [7] [8] and showed that the amplitude of the resultant current for multi junction increases with the increasing number of junctions. In a recent paper [9], we have shown that the resultant current for multi junctions gives interference and diffraction phenomena analogous to optics. The Josephson junction is considered as an electronic analogue and behaves like an inductor and capacitor. This behavior is not true only for single Josephson junction but also for multi junctions. The behavior of the multi junction can be described with different parameters (viz., inductance, capacitance, etc.), called the characteristic parameters of the Josephson junction. In this paper, we have derived the mathematical expression for inductance and capacitance of the multi junction classically, using simple mathematical techniques. The physical dimension of the Josephson junction is arbitrarily chosen. In Section 2, we have given a theoretical background of the model of multi Josephson junction. In Section 3, we have calculated the inductance and capacitance for single junction and then for multi junction separately. In Section 4, the numerical result and discussion have been carried out. Finally, the conclusion which is given in Section 5.

\section{Theoretical Background}

Josephson [1] [2] predicted that at a finite applied voltage $V$, an alternating current flows between two superconductors separated by an insulating layer and the supercurrent flow the relation

$$
I=I_{0} \sin \phi(t),
$$

where $I_{0}$ is the maximum supercurrent and $\phi(t)$ is the time dependent phase difference between the superconductors and follow the relation

$$
\mathrm{d} \phi / \mathrm{d} t=2 e V / \hbar=\omega_{J} .
$$

Let us first consider two Josephson junctions connected in parallel with an applied voltage $V$, as shown Figure 1 .

Now, the mathematical expressions for current $I_{1}$ and $I_{2}$ flowing through the Josephson junctions 1 and 2, can be written as [7] [8] [9]

$$
I_{1}=I_{01} \mathrm{e}^{i\left(\omega_{J} t+\phi\right)}
$$

and

$$
I_{2}=I_{02} \mathrm{e}^{i\left(\omega_{J} t+2 \phi\right)}
$$




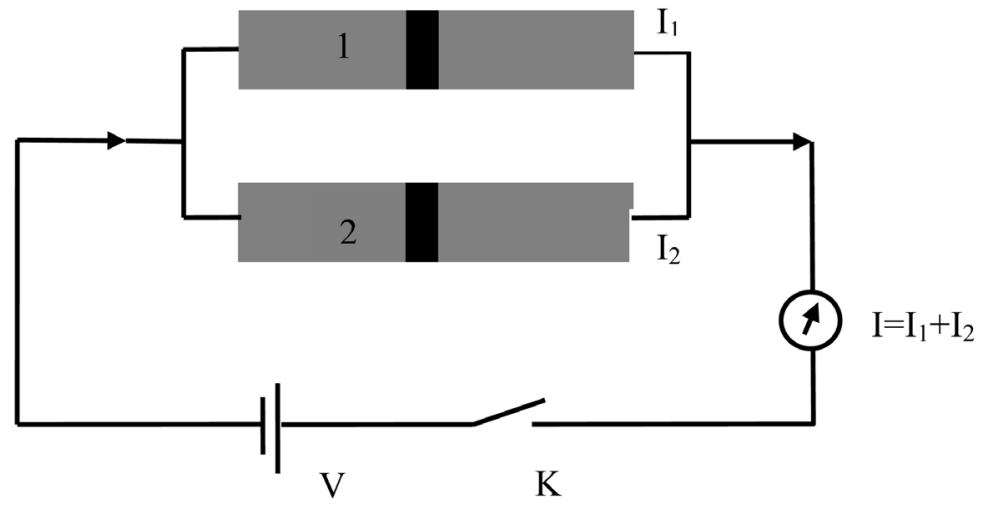

Figure 1. Schematic diagram of two parallel Josephson junctions with a constant dc voltage $(V)$.

where, $I_{01}$ and $I_{02}$ be the maximum current flowing through the junctions 1 and 2, respectively; $\phi$ and $2 \phi$ are arbitrarily chosen for the time independent phase differences across the junctions 1 and 2, respectively.

The resultant current can be written as

$$
I=I_{01} \mathrm{e}^{i\left(\omega_{J} t+\phi\right)}+I_{02} \mathrm{e}^{i\left(\omega_{J} t+2 \phi\right)}=\left(I_{01} \mathrm{e}^{i \phi}+I_{02} \mathrm{e}^{2 i \phi}\right) \mathrm{e}^{i \omega_{J} t}
$$

Considering identical junction $\left(I_{01}=I_{02}=I_{0}\right)$ and after some mathematical exercises, the resultant current due to ac Josephson effect for two junctions can be expressed as:

$$
I=I_{0} \mathrm{e}^{i \omega_{J} t}\left(\mathrm{e}^{i \phi}+\mathrm{e}^{2 i \phi}\right)=2 I_{0} \cos (\phi / 2) \sin \left(\omega_{J} t+3 \phi / 2\right)
$$

For convenience, we have taken imaginary part of the resultant current. The above formulation can be extended for more than two junctions. Let us proceed for $N$ number of identical Josephson junctions; the resultant current can be written as

$$
I=I_{0}\left[\mathrm{e}^{i\left(\omega_{J} t+\phi\right)}+\mathrm{e}^{i\left(\omega_{J} t+2 \phi\right)}+\cdots+\mathrm{e}^{i\left(\omega_{J} t+N \phi\right)}\right]
$$

Using some mathematical calculations, we have

$$
I=I_{0} \frac{\sin (N \phi / 2)}{\sin (\phi / 2)} \sin \left(\omega_{J} t+(N+1) \phi / 2\right)
$$

This is the resultant current for multi junction ac Josephson effect in superconductor. It can be noted that for $N=2$, the Equation (7) resembles to Equation (5) for two identical Josephson junctions in parallel connection, which supports our assumption.

\section{Inductance and Capacitance of Multi Josephson Junction}

\subsection{Inductance for Multi Junction ac Josephson Effect}

Let us start with a single junction in presence of applied voltage $V$, an alternating supercurrent of frequency $\omega_{J}$ flows through the junction which is given by

$$
I=I_{0} \mathrm{e}^{i\left(\omega_{J} t+\phi\right)},
$$


where the frequency of alternating supercurrent is $\omega_{J}=(2 \mathrm{eV} / \hbar)$ and $\phi$ is the phase difference, independent of time. Differentiating above current equation with respect to time and using the classical argument $V=L(\mathrm{~d} I / \mathrm{d} t)$; we have

$$
V=L\left(i \omega_{J}\right) I_{0} \mathrm{e}^{i\left(\omega_{J} t+\phi\right)}
$$

Putting $\omega_{J}=2 \mathrm{eV} / \hbar$ for single junction and taking imaginary parts as before, we have

$$
L_{J}=\frac{L_{0}}{\cos \left(\omega_{J} t+\phi\right)},
$$

where $L_{0}=\left(\hbar / 2 e I_{0}\right)$ is called the parametric inductance.

Therefore, the inductance of an ac Josephson junction is a periodic function of phase and frequency.

Now we proceed for the calculation of inductance due to 2 Josephson junctions connected in parallel (as shown in Figure 1). We use Equation (5) for the resultant current.

The corresponding inductance is found to be

$$
L_{J}=\frac{L_{0}}{2 \cos (\phi / 2) \cos \left(\omega_{J} t+3 \phi / 2\right)} .
$$

In a similar way, we proceed for $\mathrm{N}$ number of identical Josephson junction, the result is as follows

$$
L_{J}=\frac{L_{0}}{\cos \left(\omega_{J} t+(N+1) \phi / 2\right) \frac{\sin (N \phi / 2)}{\sin (\phi / 2)}} .
$$

This is the mathematical expression for inductance of the multi Josephson junction connected in parallel.

\subsection{Capacitance for Multi Junction ac Josephson Effect}

The concept of capacitance for Josephson junction is analogously defined as the capacitance of a parallel plate capacitor. A Josephson junction consists of two superconductors separated by an insulating layer, the layer may be oxide or dielectric material. Any two adjacent conductors can function as a capacitor if the charges on the conductors are $+q$ (for hole pair) and $-q$ (for electron pair). The applied voltage between the conductors gives capacitance, written as

$$
C=I\left(\frac{\mathrm{d} V}{\mathrm{~d} t}\right)^{-1}
$$

where the supercurrent flowing through the Josephson junction is given in Equation (8).

Compare to the ordinary frequency of oscillation $\omega=\sqrt{1 / L C}$ and using the inductance $L_{0}=L \equiv\left(\hbar / 2 e I_{0}\right)$, we have

$$
V=\sqrt{\left(\frac{I_{0} \hbar}{2 e C}\right)}
$$


Using $(C)^{-1} \int I \mathrm{~d} t=V$ and $I=I_{0} \mathrm{e}^{i\left(\omega_{J} t+\phi\right)}$ for single Josephson junction, the capacitance is found to be

$$
C_{J}=\frac{2 e I_{0}}{\hbar \omega_{J}^{2}} \cos ^{2}\left(\omega_{J} t+\phi\right)
$$

For 2 junctions connected in parallel (shown in Figure 1), the resultant capacitance is found to be

$$
C_{J}=\frac{8 e I_{0}}{\hbar \omega_{J}^{2}} \cos ^{2} \frac{\phi}{2} \cos ^{2}\left(\omega_{J} t+\frac{3 \phi}{2}\right) .
$$

In a similar way, for $N$ identical Josephson junction connected in parallel, the resultant capacitance is found to be

$$
C_{J}=\frac{2 e I_{0}}{\hbar \omega_{J}^{2}} \frac{\sin ^{2}(N \phi / 2)}{\sin ^{2}(\phi / 2)} \cos ^{2}\left(\omega_{J} t+(N+1) \phi / 2\right) .
$$

This is the expression for capacitance of the multi junction ac Josephson effect in superconductor.

\section{Numerical Results and Discussion}

The numerical work has been carried out for the sample HgTe based Josephson junction [10]. For numerical analysis, we use the published values of $I_{0}=2 \times 10^{-7} \mathrm{~A}$ and $\omega_{J}=3 \mathrm{GHz}$ respectively. We have taken some numerical results for inductance and capacitance in terms of frequency and phase shown in Figures 2-5. In Figure 2, we have depicted the variation of inductance with frequency for four different junctions $(N=1,2,3,4)$ and the result show the similar behavior of equivalent or kinetic inductance [11]. Figure 3 shows the variation of capacitance with frequency for the different number of junctions $(N=1$,

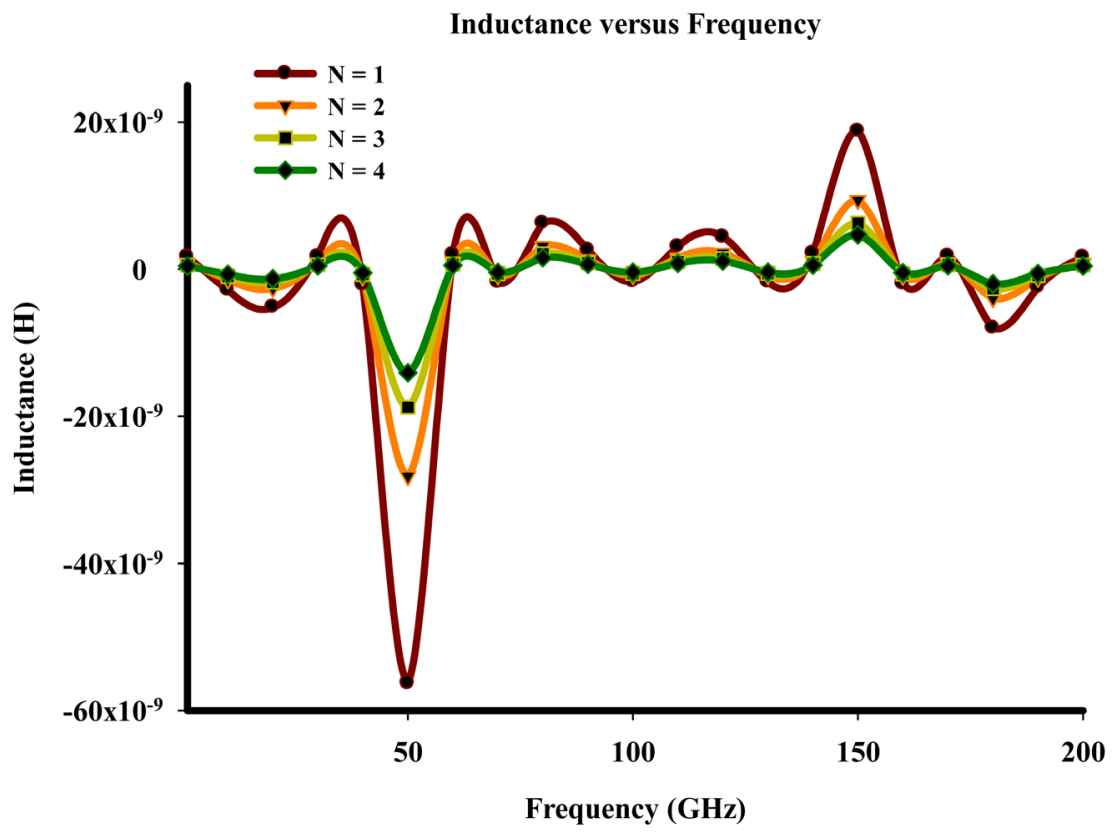

Figure 2. Plot of Inductance versus Frequency at constant phase. 


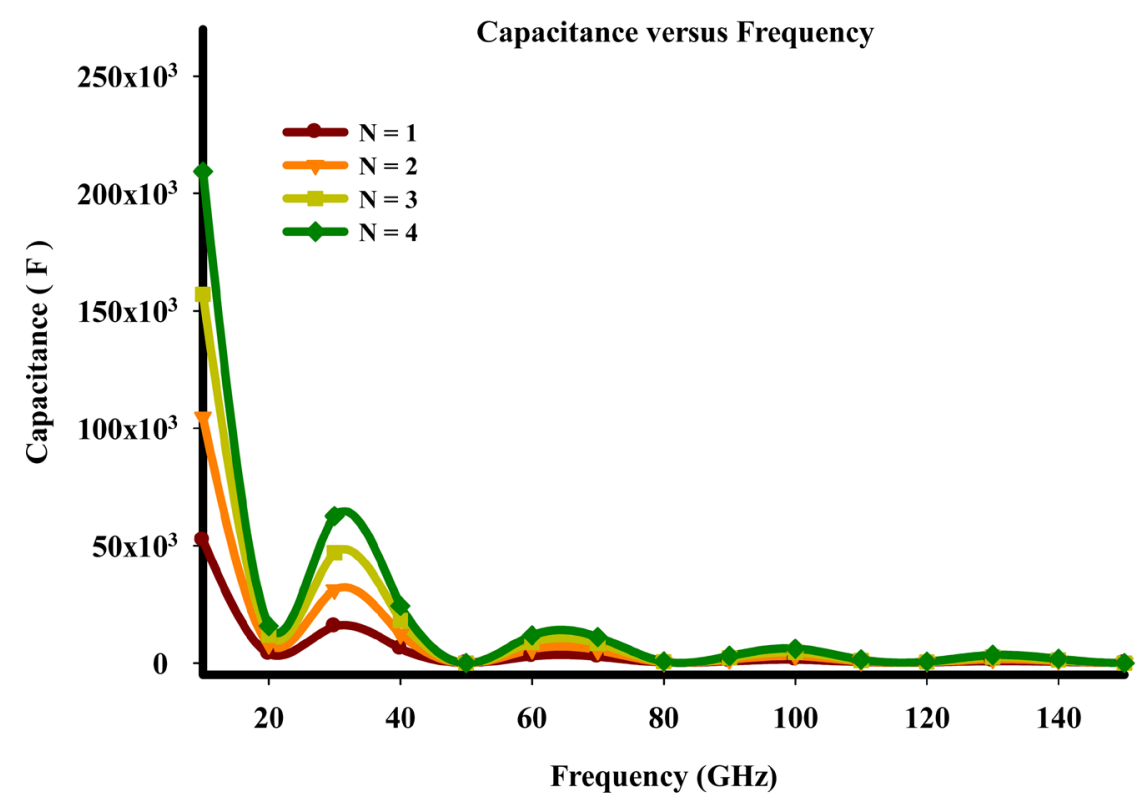

Figure 3. Plot of Capacitance versus Frequency at constant phase.

Inductance versus Phase

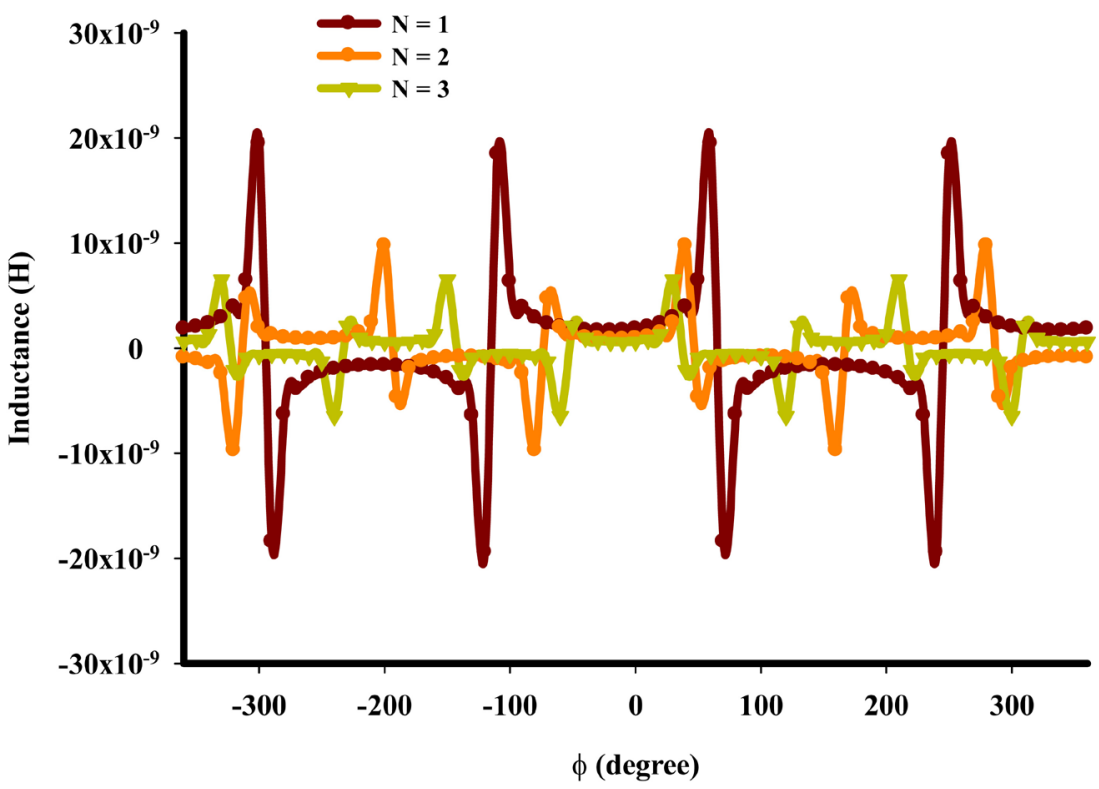

Figure 4. Plot of Inductance versus Phase.

$2,3,4)$. Here, we see that the capacitance increases with the increasing number of junctions and decreases with frequency. The result is comparable with the experimental results discussed in references [12] [13] [14] [15] and references therein. In Figure 4, we have shown the variation of inductance with phase for the different number of junctions $(N=1,2,3)$. The result is comparable with the experimental results shown in reference [16], analogous to transient (like ON/OFF peak) effect. In Figure 5, we have depicted the variation of capacitance 


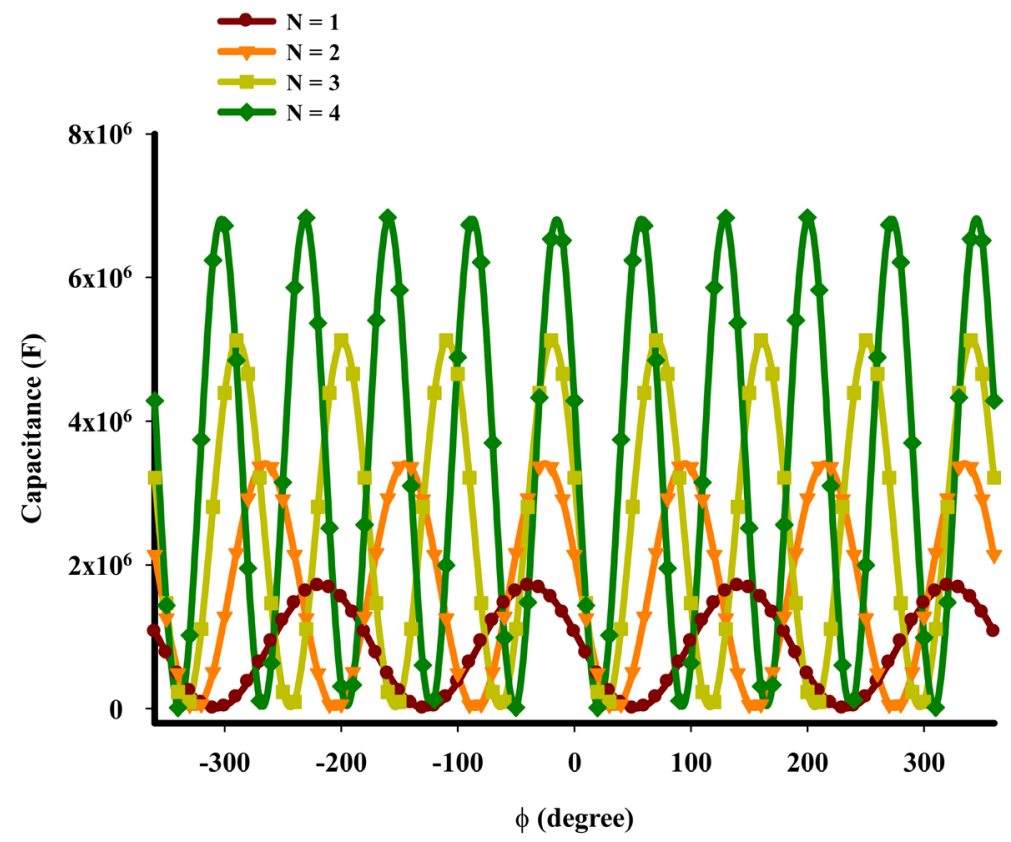

Figure 5. Plot of Capacitance versus Phase.

with phase for the different number of junctions $(N=1,2,3,4)$. Here, we see that the amplitude of the capacitance increases with the increasing number of junction and vary periodically. The periodic variations of the capacitance indicate that the multi junction can be used as a local oscillator.

\section{Conclusion}

We have studied the model of multi Josephson junction in superconductivity. The theoretical investigation based on numerical analysis of the characteristic parameters of multi Josephson junction indicates few technological advantages, like high frequency oscillator, interferometer, and qubit etc. already mentioned before. This is the first time we have evaluated the inductance and capacitance of the multi Josephson junction using simple classical argument. Addition of the multi Josephson junction model gives kinetic inductance which is highly nonlinear. The periodic variation of the kinetic inductance, i.e., positive and negative inductance indicates the simultaneous energy received and released. It is well known that the nonlinearity of the Josephson junction breaks the degeneracy of the energy states, and picks up only the two qubit states simultaneously [17]. This is the reason that we want to know how multi junction be used for qubit construction. Addition of multi junction raises the question [7] [8] [9], what types of qubit behavior it would show whether it is flux or phase qubit. The present study indicates that multi Josephson junction (connected in parallel) behaves like phase qubit. Then the evaluation of the Josephson energies for multi junction in terms of inductive energy and capacitive energy is necessary to clarify the mentioned problem which is our future study. However, we have used 
classical argument to explain the quantum behavior of multi Josephson junction in superconductivity.

\section{Acknowledgements}

The authors are grateful to the authority of Chittagong University of Engineering and Technology (CUET), Chittagong 4349, Bangladesh for all sorts of support during this work.

\section{Conflicts of Interest}

The authors declare no conflicts of interest regarding the publication of this paper.

\section{References}

[1] Josephson, B.D. (1962) Possible New Effects in Superconductive Tunneling. Physics Letters, 1, 251-253. https://doi.org/10.1016/0031-9163(62)91369-0

[2] Barone, A. and Paterno, G. (1982) Physics and Applications of the Josephson Effect. John Wiley and Sons, New York. https://doi.org/10.1002/352760278X

[3] De Luca, R. (2015) Quantum Interference in Josephson Junctions. Journal of Modern Physics, 6, 526-535. https://doi.org/10.4236/jmp.2015.65057

[4] Lucci, M., Badoni, D., Corato, V., Merlo, V., Ottaviani, I., Salina, G., Cirillo, M., Ustinov, A.V. and Winkler, D. (2016) 1D Josephson Quantum Interference Grids: Diffraction Patterns and Dynamics. Journal of Physics D: Applied Physics, 49, Article ID: 065303. https://doi.org/10.1088/0022-3727/49/6/065303

[5] Poole, C., Farach, H., Creswick, R. and Prozorov, R. (2014) Superconductivity. Elsevier, Amsterdam.

[6] Tsujimoto, M., Maeda, Y., Kambara, H., Elarabi, A., Yoshioka, Y., Nakagawa, Y., Wen, Y., Doi, T., Saito, H. and Kakeya, I. (2015) Terahertz emission from a Stack of Intrinsic Josephson Junctions in $\mathrm{Pb}$-Doped $\mathrm{Bi}_{2} \mathrm{Sr}_{2} \mathrm{CaCu}_{2} \mathrm{O}_{8+\delta}$. Superconductor Science and Technology, 28, Article ID: 105015. https://doi.org/10.1088/0953-2048/28/10/105015

[7] Islam, M.R. and Rahman, H. (2014) A Model of the Multijunction ac Josephson Effect in a Superconductor. Turkish Journal of Physics, 38, 73-80. https://doi.org/10.3906/fiz-1305-5

[8] Islam, M.R., Kabir, M.A. and Razon, M.A. (2016) Aspects of Multi-Junction ac Josephson Effect in Superconductor. International Journal of Integrated Sciences \& Technology, 2, 39-44. http://www.cuet.ac.bd/IJIST/index.html

[9] Maruf, H.M.A.R., Islam, M.R. and Chowdhury, F.-U.-Z. (2018) Analogy between ac Josephson Junction Effects and Optical Phenomena in Superconductors. Bangladesh Journal of Physics, in press.

[10] Deacon, R.S., Klapwijk, T.M., Tarucha, S., Wiedenmann, J., Leubner, P., Ishibashi, K., Bocquillon, E., Brune, C., Buhmann, H., Dominguez, F., Hankiewicz, E.M. and Molenkamp, L.W. (2016) Josephson Radiation from Gapless Andreev Bound States in HgTe-Based Topological Junctions. arXiv:1603.09611v2.

[11] Shimazu, Y. and Yokoyama, T. (2004) Measurement of Kinetic Inductance of Superconducting Wires and Application for Measuring Flux State of Josephson-Junction Loops. Physica C: Superconductivity, 412-414, 1451-1454. 
https://doi.org/10.1016/j.physc.2003.11.085

[12] Xu, P., Gu, T., Cao, Z., Wei, B., Yu, J., Li, F., Byun, J.-H., Lu, W., Li, Q. and Chou, T.-W. (2013) Carbon Nanotube Fiber Based Stretchable Wire-Shaped Supercapacitors. Advanced Energy Materials, 4, Article ID: 1300759.

[13] Zhu, J., Xu, Y., Wang, J., Lin, J., Sun, X. and Mao, S. (2015) The Effect of Various Electrolyte Cations on Electrochemical Performance of Polypyrrole/RGO Based Supercapacitors. Physical Chemistry Chemical Physics, 17, 28666-28674. https://doi.org/10.1039/C5CP04080A

[14] Sathyamoorthi, S., Kanagaraj, M., Kathiresan, M. and Velayutham, D. (2016) Ethyl Viologen Dibromide as a Novel Dual Redox Shuttle for Supercapacitor. Journal of Materials Chemistry A, 4, 4562-4569. https://doi.org/10.1039/C6TA00858E

[15] Arunkumar, M. and Paul, A. (2017) Importance of Electrode Preparation Methodologies in Supercapacitor Applications. ACS Omega, 2, 8039-8050. https://doi.org/10.1021/acsomega.7b01275

[16] Paila, A., Gunnarsson, D., Sarkar, J., Sillanpää, M.A. and Hakonen, P.J. (2009) Current-Phase Relation and Josephson Inductance in a Superconducting Cooper-Pair Transistor. Physical Review B, 80, Article ID: 144520. https://doi.org/10.1103/PhysRevB.80.144520

[17] Wendin, G. and Shumeiko, V.S. (2007) Quantum Bits with Josephson Junctions. Low Temperature Physics, 33 724-744. https://doi.org/10.1063/1.2780165 